\title{
APLICAÇÃO DE MODELO MATEMÁTICO VISANDO Ȧ ESCOLHA DA MELHOR COMBINAÇÃO DE INSTRUMENTOS PARA A DESCOBERTA E TRATAMENTO DE CASOS DE TUBERCULOSE PULMONAR *
}

\begin{abstract}
ARANTES, G. R. Aplicação de modelo matemático visando à escolha da melhor combinação de instrumentos para a descoberta e tratamento de casos de tuberculose pulmonar. Rev. Saúde públ., $S$. Paulo, 12: $455-70,1978$.
\end{abstract}

RESUMO: Com o objetivo de verificar a maneira mais eficaz para descobrir e tratar casos de tuberculose pulmonar, um modelo matemático foi aplicado $\dot{a}$ demanda do Centro de Saúde de Ribeirão Preto, São Paulo (Brasil). As 30.855 pessoas residentes com 15 ou mais anos de idade que se matricularam entre maio de 1973 e abril de 1974 foram submetidas ao teste tuberculinico, abreugrafia e exame bacteriológico do escarro (nos sintomáticos). Os resultados forneceram a composiçāo epidemiológica da demanda, constituida por cinco categorias mutuamente exclusivas: 1 - náo infectado: $61,14 \% ; 2$ - infectados: $37,84 \% ; 3-$ suspeitos $\grave{a}$ abreugrafia: $0,83 \% ; 4-$ casos positivos apenas à cultura: $0,07 \% ; 5$ - casos baciloscopia-positivos: $0,12 \%$. Paralelamente calcularam-se os custos médios unitários dos exames e do tratamento no Centro de Saúde. Com esses dados, o modelo matemático proposto por Revelle e Male, modificado neste trabalho, foi aplicado para o cálculo do custo por caso ativo tratado segundo todas as combinaçóes lógicas possiveis dos exames citados. A estratégia mais adequada à demanda estudada, nas condiçóes administrativas vigentes, definida por critérios econômico, epidemiológico e clínico foi a seguinte: inicialmente teste tuberculinico em toda a demanda; a seguir, baciloscopia de escarro apenas nos reatores, complementada por cultura dos escarros negativos; tratamento dos positivos $\dot{a}$ bacteriologia. Sendo disponivel a abreugrafia, este exame poderá ser encaixado nesta seqüência supra, entre o teste tuberculinico e a baciloscopia.

UnItermos: Epidemiologia, métodos. Tuberculose pulmonar.

A opção entre as técnicas disponiveis para o controle da tuberculose, e suas combinações, deve se basear em múltiplos critérios, entre os quais as caracteristicas epidemiológicas da área, fatores sócio-culturais, econômicos, administrativos e polí-

* Extraido da Tese apresentada à Faculdade de Saúde Pública da USP, no concurso cie Livre-docência junto ao Departamento de Epidemiologia, fevereiro, 1978.

* Da Divisão de Estudos e Programas da Coordenadoria de Saúle da Comunidade da Secretaria da Saúde do Estado de São Paulo. Av, São Luiz, $99-9$ andar. São Paulo, SP Brasil. 
ARANTES, G. R. Aolicação de modelo matemático visando à escolha da melhor combinação de instrumentos para a descoberta e tratamento de casos de tuberculose pulmonar. Rev. Saúde públ., S. Paulo, 12:455-70, 1978.

ticos, como bem o demonstraram diversos autores $0,11,17,25$.

Da mesma forma, a escolha da melhor combinação de instrumentos para a descoberta e tratamento de casos também deve ser feita a partir do conhecimento de múltiplas variáveis.

O teste tuberculínico negativo, devido ao seu elevado valor predictivo 24 , permite excluir a tuberculose doença em adultos com uma probabilidade de $99,98 \% 3$; o exame abreugráfico mostra um valor predictivo positivo que pode ser substancialmente aumentado pela triagem tuberculínica, com economia de até $65 \%$ de pessoas a exa$\operatorname{minar}^{3}$

O exame bacterioscópico de uma só amostra de escarro pode descobrir até $66 \%$ dos casos existentes"; a sensibilidade chega a alcançar $71 \%$ se forem examinadas amostras colhidas em duas manhãs consecutivas ${ }^{7}$. Entre os exames passiveis de aplicação em massa, a cultura de escarro é o mais sensivel, sendo mesmo utilizada como padrão para aferir as qualidades dos exames precedentes ${ }^{18}$.

Una vez que os recursos econômicos estão sempre sujeitos a restriçōes orçamentárias é preciso encontrar um modo de otimizar o uso desses instrumentos, isolados ou em combinações. A alocação de recursos para o tratamento de casos deve levar em conta os gastos realizados com a busca dos mesmos bem como os gastos com o tratamento propriamente dito, pois os orçamentos são propostos para todo o programa cujo objetivo final é tratar os casos e não apenas descobri-los. Essa otimização talvez seja alcançada aplicando-se o modelo proposto por Revelle e Male ${ }^{19}$, baseado na possibilidade de se utilizar os quatro exames citados - teste tuberculínico, abreugrafia, cultura e baciloscopia. Esses autores assumiram o pressuposto de que, nessa ordem, esses exames detectam a tuberculose em estágios progressivamente mais evoluídos; assim, uma pessoa abreugraficamente suspeita para tuberculose seria também tuberculino-positiva mas uma pessoa tuber- culino-positiva não teria que ser necessariamente suspeita ao exame abreugráfico; uma cultura positiva implicaria abreugrafia suspeita mas não obrigatoriamente em baciloscopia positiva; esta, por sua vez, implicaria em positividade de todos os outros exames. Aceitos esses pressupostos, que na prática de saúde pública raramente deixam de prevalecer, uma população alvo submetida aos exames em pauta pode ser dividida em cinco categorias mutuamente exclusivas: não infectados, infectados, suspeitos à abreugrafia, casos cultura-positivos e casos baciloscopia-positivos. $O$ tamanho relativo dessas categorias variará de uma área para outra em função das características epidemiológicas da tuberculose.

Aos membros da população alvo, toda ela, amostra ou demanda, poderá ser oferecido tratamento sem qualquer exame prévio, com o que seguramente todos os casos nela contidos serão atingidos, embora a um custo exorbitante; ou então apenas aos tuberculino-positivos, a um custo bem menor, embora assim muitos não-doentes também fossem tratados desnecessariamente; ou qualquer combinação de dois, três ou quatro exames precedendo o tratamento, com 0 que se aumentam as probabilidades de tratar apenas as pessoas doentes. Quanto maior o número de exames efetuados antes do tratamento, maior o custo do componente diagnóstico do programa e presumivelmente menor o custo do tratamento; quanto mais eficaz a fase diagnóstica, mais casos serão descobertos, diminuindo a participação dos gastos com o diagnóstico na formação do custo médio por caso tratado. As diferentes alternativas podem ser delineadas por meio de uma "árvore de decisões" cujos ramos são constituídos pelas diferentes sequiências lógicas de exames e tratamento, representados por fórmulas matemáticas simples ${ }^{19}$. Sua aplicação mostrará a seqüência capaz de fornecer 0 menor custo por caso ativo tratado (CCAT). A combinação ideal dependerá do custo unitário de cada exame, do custo do tratamento e da magnitude das categorias 
ARANTES. G. R. Aplicação de modelo matemático risando à escolha da melhor combinação de instrumentos para a descoberta e tratamento de casos de tuberculose pulmonar. Rer. Saúde publ., S. Paulo. 12:455-70. 1978.

epidemiológicas da tuberculose na população alvo. Consequientemente, o custo médio por caso ativo tratado poderá ser diferente de uma regiāo para outra ou de uma para outra demanda de uma mesma área.

O presente trabalho foi planejado com us seguintes objetivos: 1 - Verificar se o modelo em tela é aplicável nas condições habituais de funcionamento de uma unidade sanitária polivalente, isto é, com um mínimo de alteraçōes na sua rotina. 2 - Determinar qual a combinação de instrumentos mais adequada à demanda estudada.

\section{MATERIAL E MÉTODOS}

A pesquisa foi realizada em Ribeirão Preto, Estado de São Paulo, onde viviam 235.231 pessoas. A população de estudo foi constituida pelas pessoas com 15 ou mais anos de idade, residentes no municipio, que se matricularam no Centro de Saúde entre 1 de maio de 1973 a 30 de abril de 1974.

Ao todo foram matriculadas 32.225 pessoas, interrogadas quanto à existência de sintomas respiratórios, abreugrafadas, submetidas ao teste tuberculínico ${ }^{6}$ e exame bacteriológico de escarro 13,14 quando portadoras de expectoração. Desse total, 30.885 voltaram para a leitura do teste $(95,8 \%)$ dos quais $1.085(3,5 \%)$ tiveram seu escarro submetido à baciloscopia e cultura para BK. Por ocasião da matrícula era preenchida uma ficha codificada, confeccionada para esta pesquisa. Em seguida as pessoas eram abreugrafadas e logo depois submetidas ao teste tuberculinico, quando recebiam orientação para a colheita do escarro e dia de retorno para a leitura do Mantoux e entrega do pote com escarro. Uma vez completados os dados, as fichas foram encaminhadas ao Centro de Processamento de Dados

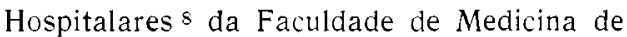
Ribeirão Preto, onde as informaçōes codificadas nelas contidas foram passadas para cartōes IBM, conferidas e apuradas mecanicamente.
Os gastos diretos com as atividades finais (saneamento, oftalmologia, hanseniase, imunizações, odontologia, assistência materno-infantil, assistência ao adulto e tisiologia) e intermediárias (direção, chefia, assessoria, medicamentos de uso geral, visitação domiciliária e administração auxiliar) foram levantados pelo Método CENDES/OPS de planejamento 1.4. A seguir, o custo total das atividades-meio foi rateado entre as atividades finais em função do valor percentual dos custos de cada uma delas, obtendo-se com isso o custo total de cada atividade fim, isto é, custos diretos mais custos indiretos. Posteriormente, usando-se o mesmo processo, calcularam-se os custos com as sub-atividados de tisiologia - cadastro tuberculinico, cadastro abreugráfico, cadastro baciloscópico, cadastro cultura e tratamento. Finalmente, o custo médio unitário por exame realizado foi obtido dividindo-se o custo total de cada cadastro pelo volume de atendimentos realizados no período de duração da pesquisa e o custo médio do tratamento, em função de todos os doentes tratados.

No modelo original todas as pessoas das várias categorias epidemiológicas citadas seriam submetidas à baciloscopia ou cultura para BK quando esses exames fizessem parte da fórmula, por meio de "swab" laringeo nos assintomáticos; como esse método é inadequado para uso em massa, além de pouquíssimo eficaz, foram realizados exames bacteriológicos apenas em pessoas portadoras de expectoração. Consequientemente foi preciso introduzir nas fórmulas fatores de correção que expressassem o verdadeiro número de pessoas submetidas à baciloscopia e/ou cultura em cada uma delas. Esses fatores decorreram da proporção de sintomáticos respiratórios (SR) que variou conforme a reatividade tuberculinica dos mesmos (Tabela 1). Os dados dessa Tabela significam que 
ARANTES. G. R. Aplicação de modelo matemátıco visando à escolha da melhor combinação de instrumentos para a descoberta e tratamento de casos de tuberculose pulmonar. Rev. Saúde públ., S. Paulo, 12:455-70, 1978.

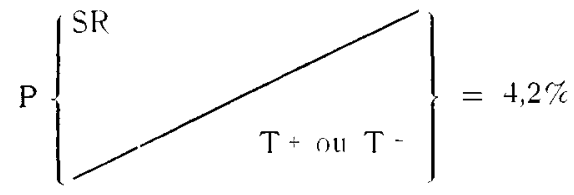

isto é, a probabilidade de uma pessoa da demanda ser sintomática respiratória dado que 0 seu teste tuberculínico tenha sido positivo ou negativo é igual a $4,2 \%$.

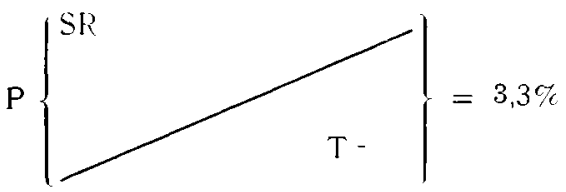

ou seja, a probabilidade de una pessoa dessa demanda ser sintomática-respiratória se o seu teste tuberculínico for negativo é igual a $3,3 \%$.

Finalmente,

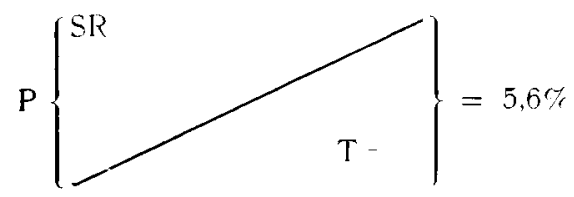

significa que a referida probabilidade, se o teste for positivo, é de $5,6 \%$.

Por isso foram introduzidos 2 fatores de correção, $\mathrm{f}_{1}=0,042(4,2 \%)$ e $\mathrm{f}_{2}=0,056$ $(5,6 \%)$.

Tratando-se de fórmulas que se iniciam pela baciloscopia ou pela cultura, devendo portanto abranger tanto $\mathrm{T}+$ como $\mathrm{T}$-, usouse $f_{1}$. Nas fórmulas em que o exame de escarro é realizado em pessoas previamente selecionadas pelo teste tuberculínico (reatores fracos ou fortes) usou-se $f_{2}$.

Nas poucas pessoas sem expectoração selecionadas pela abreugrafia justifica-se a coleta de material para exame pelo "swab" laringeo e nesse caso considerou-se que todas poderiam ser examinadas bacteriologicamente, dai a não necessidade de fator de correção. Finalmente, não se usou o fator para os tuberculino-negativos porque esses são de início descartados em todas as seqüências.

Conhecidos os custos unitários do teste tuberculínico $\left(C_{t}\right)$, da abreugrafia $\left(C_{r}\right)$, da baciloscopia $\left(C_{b}\right)$, da cultura $\left(C_{c}\right)$ e do tratamento $(F)$, e calculadas as frações (frações de um) da população de demanda em cada uma das categorias epidemiologicas - não infectados, infectados (I), suspeitos à abreugrafia (S), casos culturapositivos (C) e casos baciloscopia-positivos (B) - , aplicaram-se as fórmulas deduzidas por Revelle e Male ${ }^{19}$, com a introdução dos fatores de correção quando indicada, apresentadas em Anexo.

\section{PESULTADOS}

A demanda estudada, isto é, pessoas que se submeteram pelo menos ao teste tuberculinico e abreugrafia, foi constituída por 30.885 pessoas. A proporção de sintomáticos respiratórios (SR) segundo o resultado do teste tuberculinico é apresentada na Tabela 1 e a composição epidemiológica da mesma face aos resultados dos exames diagnósticos de aplicação em massa pode ser observada na Tabela 2. A discrepância entre o número de não-reatores contidos nessas Tabelas será explicada na discussão.

Os gastos* com atividades intermediárias (direção, assessoria, administração), incluindo pessoal e material, atingiram a cifra de $\operatorname{Cr} \$ 706.926,00$ ao passo que os gastos diretos com as atividades finais somaram Cr\$ 1.993.988,00, sendo que as atividades de tisiologia participaram com $18,49 \%$ desse total $(\operatorname{Cr} \$ 368.678,00)$. Os gastos diretos com as sub-atividades de tisiologia foram de Cr $\$ 8.000,00$ com o cadastro tuberculínico, de $\mathrm{Cr} \$ 73.018,00 \mathrm{com}$

\footnotetext{
* Para se fazer o célculo do valor equivalento em dolar. calcular a quantia de Cr\$ 6.30 para cada dolar.
} 
ARANTES, G. R. Aplicação de modelo matemático visando à escolha da melhor combinação de instrumentos para a descoberta e tratamento de casos de tuberculose pulmonar. Rev. Saúde públ., S. Paulo, 12:455-70, 1978.

T A B E L A 1

Proporção (\%) de sintomáticos respiratórios *, segundo o resultado do teste tuberculínico, residentes com 15 anos e mais de idade, Centro de Saúde de Ribeirão Preto, SP, 1973/74.

\begin{tabular}{lccc}
\hline & Total de cadastrados & \multicolumn{2}{c}{ Sintomáticos respiratórios } \\
\cline { 3 - 4 } Resultado & & $\mathrm{N} \%$ & $\%$ \\
\hline Não-reatores & 18.948 & 633 & 3,3 \\
Reatores (fracos e fortes) & 11.937 & 671 & 5,6 \\
\hline Total & 30.885 & 1.304 & 4,2 \\
\hline
\end{tabular}

* Incluídos os não-examinados por motivos explicados em trabalho de Arantes e Trivellato 5, 1976.

T A B E L A 2

Categorias epidemiológicas da demanda estudada, em função dos resultados dos exames para diagnóstico aplicados em massa, em números absolutos e frações de um, residentes, com 15 anos e mais de idade, Centro de Saúde de Ribeirão Preto, SP, 1973/74.

\begin{tabular}{lrr}
\hline Categoria epidemiológica & No & Fração de 1 \\
\hline $1-$ Não-infectados & 18.883 & 0,6114 \\
$2-$ Infectados sem abreugrafias suspeitas & 11.688 & 0,3784 \\
$3-$ Infectados, suspeitos, bacteriologicamente negativos & 257 & 0,0083 \\
$4-$ Infectados, suspeitos, apenas cultura-positivos & 20 & 0,0007 \\
5 - Infectados, suspeitos, cultura e baciloscopia-positivos & 37 & 0,0012 \\
\hline Total & 30.885 & 1,0000 \\
\hline
\end{tabular}

o cadastro abreugráfico, de Cr\$ $6.727,00$ com o cadastro baciloscópico, de Cr\$ ... $17.577,00 \mathrm{com}$ o cadastro cultura e Cr\$ $263.356,00$ com o tratamento. O rateio dos $18,49 \%$ dos custos intermediários, ou seja, de $\operatorname{Cr} \$ 130.710,00$ entre as respectivas subatividades de tisiologia e a posterior divisão dos custos totais (diretos + indiretos) de cada uma delas pelo número total de pessoas examinadas forneceu os seguintes custos médios unitários por pessoa examinada . teste tuberculínico $\mathrm{Cr} \$ 0,30$, abreugrafia $\operatorname{Cr} \$ 2,00$, baciloscopia $\operatorname{Cr} \$ 8,40$ e cultura Cr $\$ 22,00$, sendo que o custo médio do tratamento foi de Cr $\$ 610,00$ por pessoa tratada. (Ressalte-se que esses custos são válidos apenas para o Centro de Saúde em tela e nas condições vigentes na época).

Conhecidas as categorias das pessoas da demanda em frações de um (Tabela 2) e os custos unitários e aplicadas as fórmulas apresentadas em Anexo, obtiveram-se os custos médios por caso ativo tratado (CCAT) de acordo com a seqüência de exames utilizada para o diagnóstico, segundo se pode notar na Tabela 3. 
ARANTES, G. R. Aplicação de modelo metamático visando à escolha da melhor combinação de instrumentos para a descoberta e tratamento de casos de tuberculose pulmonar. Rev Saúde públ., S. Paulo, 12:455-70, 1978.

\section{T A B E L A 3}

Custo por caso ativo (de tuberculose pulmonar) tratado, de acordo com a seqüência de exame para o diagnóstico, na demanda local com 15 anos e mais de idade, Centro de Saúde de Ribeirão Preto, 1973/1974.

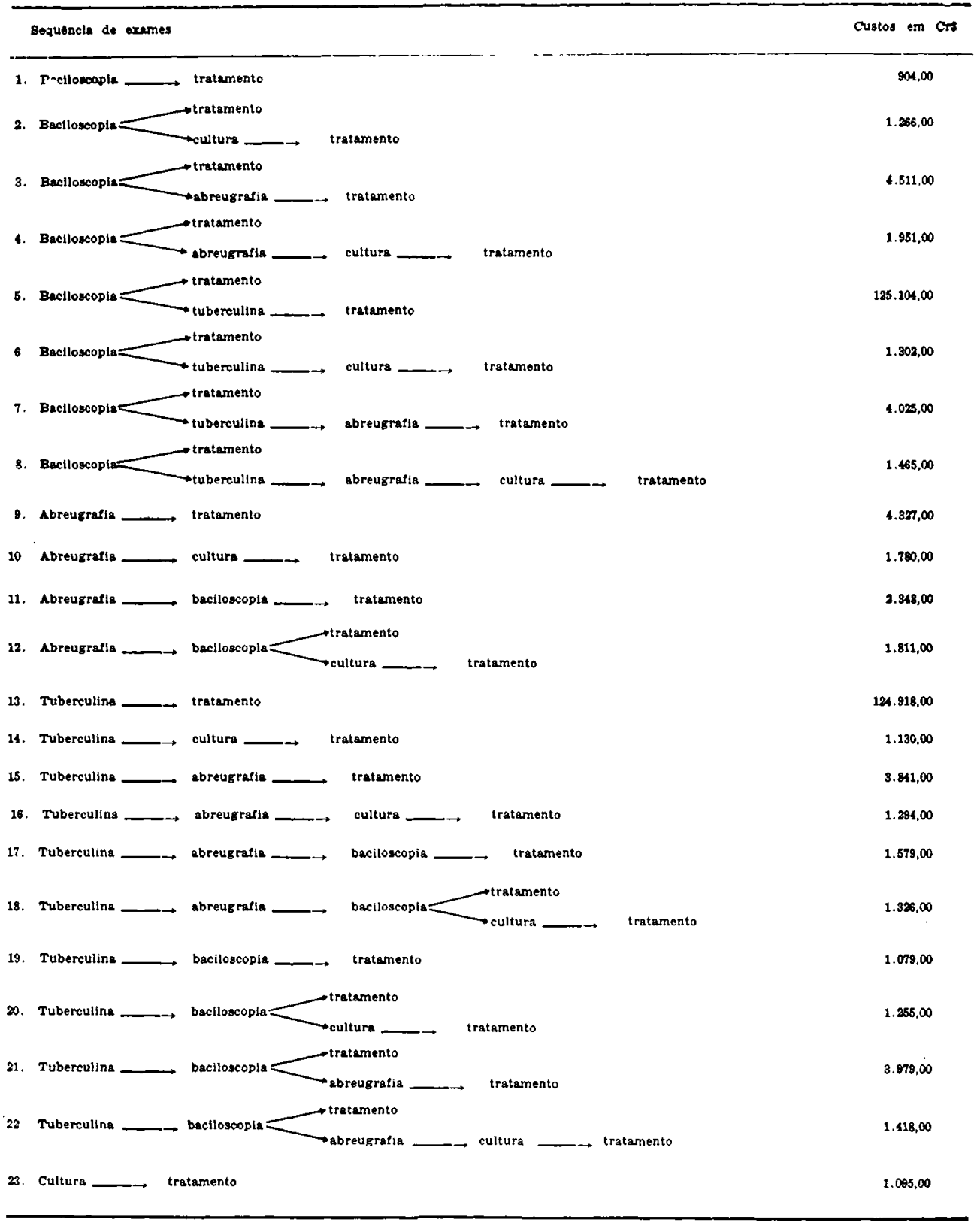


ARANTES, G. R. Aplicação de modelo matemático visando à escolha da melhor combinação de instrumentos para a descoberta e tratamento de casos de tuberculose pulmonar. Rev. Saúde públ., S. Paulo, 12:455-70, 1978.

\section{DISCUSSĀO}

Aproximadamente $20 \%$ da população residente no município, com 15 anos e mais de idade, demandou ao Centro de Saúde, durante $o$ ano de duração da pesquisa, sendo que $13,5 \%$ o fizeram por se sentirem doentes, e as demais para a obtenção de "certificados de saúde". Mais da metade da demanda $(53 \%)$ foi constituída por pessoas com idade variando de 15 a 25 anos e apenas $6,6 \%$ constituída por maiores de 50 anos.

Prevendo dificuldades na obtenção de duas amostras consecutivas de escarro, como se verificou alhures ${ }^{21}$, foi solicitada uma só amostra de cada sintomático. A colheita de duas amostras em dias consecutivos é mais eficaz quando se faz apenas baciloscopia ${ }^{14}$; tratando-se de baciloscopia seguida de cultura nos negativos, a eficácia é bem mais elevada mesmo com uma só amostra ${ }^{7}$. Como foi mencionado, a classificação das pessoas em cinco categorias epidemiológicas baseou-se na premissa de que, na ordem apresentada, os exames utilizados diagnosticam a tuberculose em estágios progressivamente mais evoluidos, caracterizando as seguintes categorias mutuamente exclusivas:

$$
\begin{aligned}
& 1-\mathrm{T}^{-}, \mathrm{R}^{-} \mathrm{C}^{-} \mathrm{B}^{-} \\
& 2-\mathrm{T}^{+}, \mathrm{R}^{-} \mathrm{C}^{-} \mathrm{B}^{-} \\
& 3-\mathrm{T}^{+} \mathrm{R}^{+} \mathrm{C}^{-} \mathrm{B}^{-} \\
& 4-\mathrm{T}^{+} \mathrm{R}^{+} \mathrm{C}^{+}, \mathrm{B}^{-} \\
& 5-\mathrm{T}^{+} \mathrm{R}^{+} \mathrm{C}^{+} \mathrm{B}^{+}
\end{aligned}
$$

Todavia, isso nem sempre acontece pois uma pessoa pode apresentar um resultado negativo para um teste menos sensivel e no entanto ser positiva para um ou mais testes dotados de maior sensibilidade; ou um resultado falso positivo para um teste pouco específico e posteriormente se mostrar positiva a um teste com maior especificidade.
Tais possibilidades, na prática, se resumem às seguintes:

$$
\begin{aligned}
& 1-\mathrm{R}^{+} \operatorname{com} \mathrm{T}^{-} \\
& 2-\mathrm{C}^{+} \text {ou } \mathrm{B}^{+} \operatorname{com} \mathrm{T}^{-} \\
& 3-\mathrm{C}^{+} \text {ou } \mathrm{B}^{+} \operatorname{com} \mathrm{R}^{-} \\
& 4-\mathrm{B}^{+} \operatorname{com~} \mathrm{C}^{-}
\end{aligned}
$$

Além dos $18.883 \mathrm{~T}$ - classificados na categoria 1 (Tabela 2), outras 65 pessoas foram $\mathrm{T}^{-}\left(64\right.$ com $\mathrm{R}^{+}$e 1 com $\mathrm{R}^{+}$e $\left.\mathrm{C}^{+}\right)$. Onde classifica-las?

a) Pessoas com $\mathrm{R}^{+}$e $\mathrm{T}^{-}$: sabendo-se que a $\%$ de falsos negativos ao teste tuberculínico (sensibilidade de 98,5\%) é semelhante à $\%$ de falsos positivos ao exame abreugráfico (especificidade de $99 \%$ ), os 64 casos $\mathrm{R}^{+} \mathrm{T}^{-}$poderiam ser indiferentemente considerados como $T-R^{-}$ ou $\mathrm{T}^{+} \mathrm{R}^{+}$. Como $\mathrm{T}^{-} \mathrm{R}^{-}$entrariam na categoria $n^{\prime \prime} 1$ à qual acresceriam apenas $0,33 \%$; porém, como $\mathbf{T}+\mathrm{R}^{+}$entrariam na categoria n" 3 onde se constituiriam em $21 \%$. Optamos pela sua inclusão na última, onde seria maior sua influência na magnitude da categoria e, conseqüentemente, no cálculo dos custos.

b) Pessoas com $\mathrm{C}^{+}$e $\mathrm{T}^{-}$: dos tuberculino-negativos 526 foram submetidos a exame de escarro sendo que um deles foi $\mathrm{R}^{+} \mathrm{C}^{+}$. Por ter apresentado dois dos três exames positivos e porque a possibilidade de ser falso $\mathrm{C}^{+}$é quase nula, foi classificado na categoria $\mathrm{n}^{0} 4\left(\mathrm{~T}+\mathrm{R}^{+} \mathrm{C}^{+}\right)$.

c) Pessoas com $\mathrm{C}^{+}$ou $\mathrm{B}^{+}$entre os $\mathrm{R}^{-}$: nāo houve nenhum caso neste material embora isso seja possível 20.

d) A possibilidade de ser $\mathrm{B}^{+}$com $\mathrm{C}^{-}$ existe, especialmente quando se trata de BK resistente à isoniazida ${ }^{16}$, ou de pacien tes em quimioterapia (o que não era o caso); no presente material isso não ocorreu, e, quando acontece, costuma-se repetir o exame bacteriológico para confirmação. 
ARANTES, G. R. Aplicação de modelo matemático visando à escolha da melhor combinação de instrumentos para a descoberta e tratamento de casos de tuberculose pulmonar. Rev. Saúde públ., S. Paulo, 12:455-70, 1978.

A demanda estudada apresentou $61,3 \%$ de não-reatores, que para fins práticos podem ser considerados como não infectados pelo M. tuberculosis.

Trata-se de um elevado contingente de pessoas, predominantemente jovens, aos quais se poderia ter fornecido atestado de saúde quanto à tuberculose com base nesse resultado, uma vez que a probabilidade dos negativos não serem tuberculosos é da ordem de $99,98 \%$.

Outro aspecto a ressaltar é o fato de $35 \%$ dos casos descobertos terem sido positivos apenas à cultura, o que sugere a utilidade desse método em áreas epidemiologicamente semelhantes; essa situação é um meio termo entre o que foi observado em outros paises, tais como a Tchecoslovaquia, Inglaterra, Bélgica e India, onde as percentagens de casos positivos apenas à cultura foram respectivamente, $65 \%, 65 \%$, $61 \%$ e $18 \% 12,15,23$.

Se compararmos os cadastros abreugráfico, baciloscópico e cultura apenas quanto aos custos por caso descoberto é possivel demonstrar a superioridade da bacteriologia sobre a abreugrafia e da baciloscopia sobre a cultura. Esse tipo de análise, entretanto, é insuficiente para uma tomada racional de decisões porque não leva em conta as várias combinações possiveis nem inclui os custos do tratamento, tal como acontece com o modelo em pauta.

Visando facilitar a análise e considerando que os Centros de Saúde podem ser divididos em duas categorias - com e sem instalações abreugráficas - os resultados das diversas combinações foram agrupados conforme a ausência ou presença desse recurso (Tabela 4 e 5 ), em ordem crescente de custo médio por caso ativo tratado. No que tange às combinações da Tabela 4 , analisando-se apenas sob critério econômico, a escolhida seria a primeira seqüência.

Acontece que as unidades sanitárias, ao submeterem seus usuários adultos a um exame seletivo para tuberculose, fazem-no tanto para descobrir casos como para for- necer atestados de normalidade em relação à essa doença (Atestados de Saúde). Ora, a simples negativa de expectoração não seria suficiente para o fornecimento desse atestado devido à ocorrência de falsos assintomáticos ${ }^{5}$. A triagem prévia pelo teste tuberculínico, cujo valor predictivo negativo é quase $100 \%$, contribuiria para contornar essa dificuldade: atestado de normal aos não-reatores; interrogatório e exame de escarro para os reatores sintomáticos. Aqui, embora um pouco mais onerosa, a segunda combinação seria preferível.

Dependendo da incidência de casos positivos apenas à cultura, sendo disponível esse exame (como é o caso do Estado de São Paulo), a sequiência ideal seria a quinta, capaz de descobrir práticamente $100 \%$ dos casos. O que, além de mais justo do ponto de vista ético (maior alívio de sofrimento humano), é mais correto epidemiologicamente, pois os casos positivos apenas à cultura também infectam seus comunicantes, especialmente os menores de 10 anos ${ }^{10,22}$; e possuem a potencialidade de evoluir para formas positivas à baciloscopia.

Tratando-se de Centro de Saúde equipado com abreugrafia (Tabela 5) nota-se que a primeira seqüência é a preferivel, do ponto de vista econômico, com o inconveniente da demora para a confirmação diagnóstica. Com a segunda sequência, apenas $2,4 \%$ mais onerosa, esse inconveniente deixa de existir. Chama a atenção o fato da sétima sequiència, que é aquela tradicionalmente usada, ser $37 \%$ mais cara que a segunda, que nada mais é que a sétima, com inserção do teste tuberculínico como instrumento de triagem para abreugrafia.

A comparação entre os custos da Tabela 4 com os da Tabela 5, confrontando-se seqüências diferentes apenas quanto à presença ou não da abreugrafia, revela que o custo médio por caso tratado foi superior naquelas da Tabela 5 (sem aumentar o número de casos ativos descobertos), como se poderá observar na Tabela 6 . 
ARANTES, G. R. Aplicação de modelo matemático visando à escolha da melhor comblnaçăo de instrumentos para a descoberta e tratamento de casos de tuberculose pulmonar. Rev. Saúde públ., S. Paulo, 12:455-70, 1978.

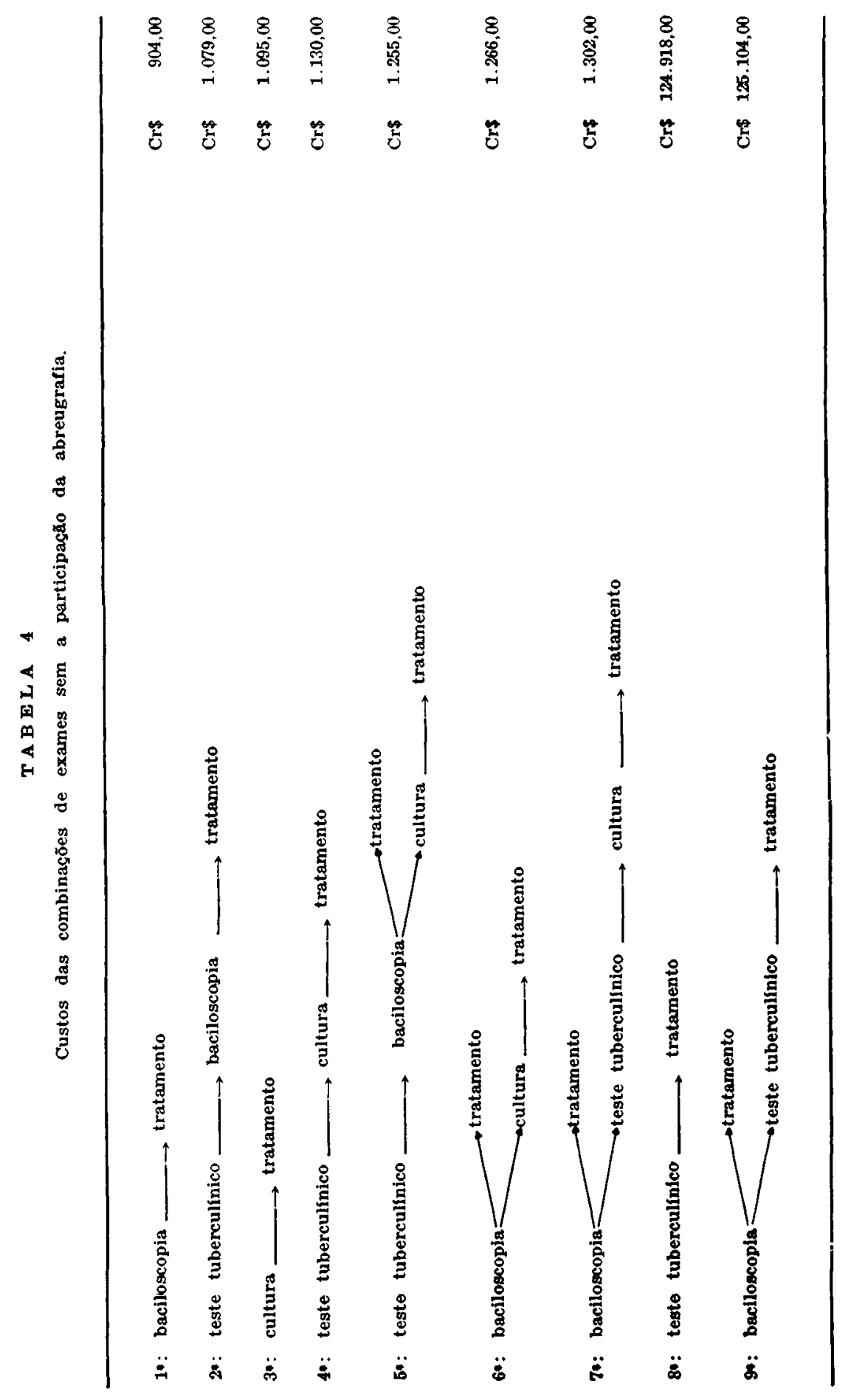


ARANTES, G. R. Aplicaçăo de modelo matemático visando à escolha da melhor combinação de instrumentos para a descoberta e tratamento de casos de tuberculose pulmonar. Rev. Saúde puibl., S. Paulo, 12:455-70, 1978.

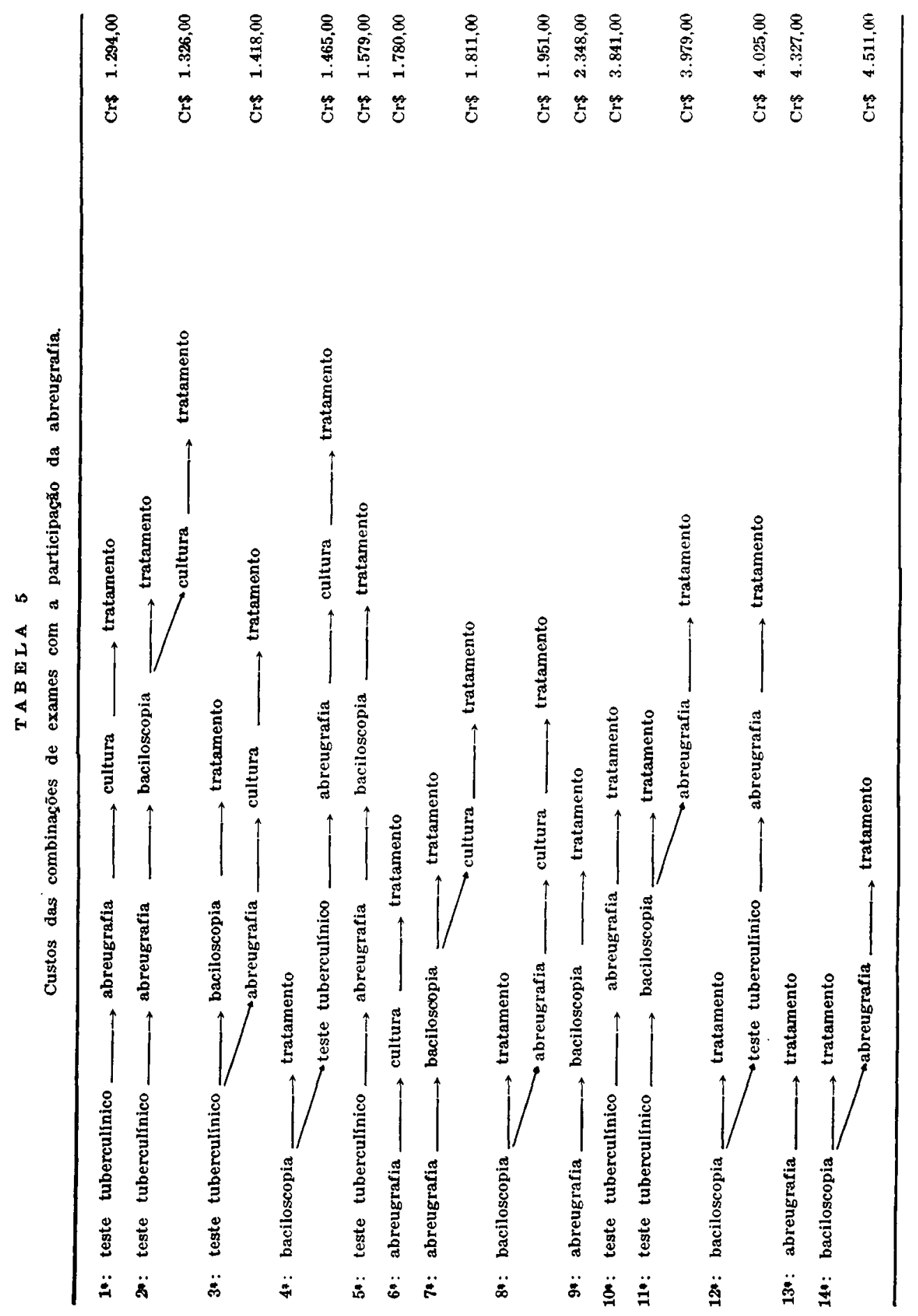


ARANTES, G. R. Aplicação de modelo matemático visando à escolha da melhor combinação de instrumentos para a descoberta e tratamento de casos de tuberculose pulmonar. Rev. Saúde públ., S. Paulo, 12:455-70, 1978.

T A B E L A 6

Comparação dos custos entre sequências diferentes apenas quanto à presença de abreugrafia.

\begin{tabular}{|c|c|c|c|c|c|c|}
\hline \multicolumn{6}{|c|}{ Custos em cruzeiros } & \multirow{3}{*}{$\begin{array}{c}\text { Encarecimento } \\
\% \\
14\end{array}$} \\
\hline \multicolumn{3}{|c|}{$\begin{array}{c}\text { sem abreugrafia } \\
\text { (Tabela 4) }\end{array}$} & \multicolumn{3}{|c|}{$\begin{array}{c}\text { com abreugrafia } \\
\text { (Tabela 5) }\end{array}$} & \\
\hline $4^{4}$ & seqüência: & $1.130,00$ & $1^{\text {月 }}$ & seqüência: & $1.294,00$ & \\
\hline $5^{4}$ & seqüência: & $1.255,00$ & $2^{*}$ & seqüência: & $1.326,00$ & 6 \\
\hline $2^{*}$ & sequiência: & $1.079,00$ & 54 & seqüência: & $1.579,00$ & 46 \\
\hline $3^{a}$ & seqüência: & $1.095,00$ & $6^{n}$ & sequência: & $1.780,00$ & 62 \\
\hline $6 a$ & seqüência: & $1.266,00$ & $7^{a}$ & sequiência: & $1.811,00$ & 43 \\
\hline 14 & seqüência: & 904,00 & $9^{4}$ & sequiência: & $2.348,00$ & 160 \\
\hline $8^{a}$ & seqüência: & $124.918,00$ & $10^{*}$ & sequiência: & $3.841,00$ & - \\
\hline
\end{tabular}

A única exceção foi a décima seqüência da Tabela 5 , que se mostrou notavelmente menos onerosa que a oitava seqüência da Tabela 4; isto porque, com esta última, todos os reatores seriam tratados como doentes ao passo que com a outra apenas os portadores de sombras suspeitas 0 serjam.

Segundo os autores do modelo, quanto maior o número de exames utilizados, maior o custo do componente diagnóstico do programa, porém menor o custo do componente tratamento, uma vez que o número de pessoas a tratar será menor.

$\mathrm{Na}$ verdade, não é apenas o número de exames utilizados, mas também os seus atributos que influirāo no número de pessoas tratadas e, conseqüentemente, nos custos; uma sensibilidade maior permite descobrir mais doentes aumentando os custos totais do programa; maior especificidade, diminuindo o número de "falsos doentes", implica diminuição das despesas com tratamento. Não é por outro motivo que as combinações cujos produtos finais decorrem do teste tuberculínico (pouco especifico quanto à tuberculose-doença) apresentam custos tão altos por caso ativo tratado $\left(8^{\mathfrak{a}}\right.$ e $9^{\mathrm{a}}$ sequiências da Tabela 4$)$.
Em contrapartida, as combinações cujo produto final decorreu da cultura do escarro, revelaram-se apenas um pouco mais onerosas que aquelas nas quais a baciloscopia determinou o produto final, apesar da cultura ser três vezes mais cara. Embora mais cara, e por isso aumentando o valor do numerador da fração, descobrindo mais doentes aumenta muito mais o denominador, com o que o quociente (custo médio por caso ativo tratado) é pouco aumentado. Obviamente em outras áreas e com outras relaçōes de custos entre esses exames as coisas poderão ocorrer de maneira diversa.

Daí ser aconselhável a aplicaçāo desse modelo, bastante simples e exequivel, para uma decisão mais acertada. Isso não implica repetição da experiência aqui apresentada; contando-se com dados aproximados quanto à composição epidemiológica da demanda e custos aproximados dos exames e do tratamento, o modelo pode ser alimentado com os mesmos, fazendo-se correçōes posteriores, à medida em que o programa se desenvolve.

\section{CONCLUSOESS}

1 - A aplicação de um modelo matemático, utilizável em condições rotineiras, per- 
ARANTES, G. R. Aplicação de modelo matemático visando à escolha da melhor combinação de instrumentos para a descoberta e tratamento de casos de tuberculose pulmonar. Rev. Saúde públ., S. Paulo, 12:455-70, 1978.

mite determinar a melhor combinação de instrumentos para a busca e tratamento de casos de tuberculose pulmonar em uma demanda.

2 - $\mathrm{Na}$ demanda estudada, as combinaçóes de instrumentos mais adequadas foram as seguintes: nas unidades sem abreugrafia, inicialmente teste tuberculínico em toda a demanda; a seguir, baciloscopia de escarro apenas nos reatores, complementada por cultura dos escarros negativos e tratamento dos positivos à bacteriologia. Sendo disponivel a abreugrafia, este exame poderá ser encaixado na seqüência supra, entre o teste tuberculínico e a baciloscopia.

RSPUB9/433

ARANTES, G. R. [Application of a mathematical model for calculating the best pattern for testing and treating a target population for pulmonary tuberculosis] Rev. Saúde públ., S. Paulo, 12:455-70, 1978.

ABSTRACT: In order to calculate the best patterns for testing and treating a target population for pulmonary tuberculosis, a mathematical model was applied to the population aged 15 and over registered at the Public Health Center of Ribeirão Preto, SP, Brazil, during 12 consecutive months, from May 1973 through April 1974. Thirty thousand eight hundred and eighty-five persons living in this area were submitted to the tuberculin test, roentgenphotography, and bacteriologic examination of sputum (symtomatic persons only). The target populatoin was divided into five non-overlapping categories according to the results of the four tests: 1 - uninfected persons, $61.14 \% ; 2$ - infected persons, $37.84 \% ; 3$ persons with lung shadows, $0.83 \% ; 4$ - only culture-positive patients, $0.07 \%$; 5 - smear-positive patients, $0.12 \%$. The average individual costs were also calculated along with cost evaluation for treatment at that Health Center tuberculin test, Cr\$ 0,30 (US\$ 0.05); roentgenphotography, Cr\$ 2,00 (US\$ 0.30); smear examination, Cr $\$ 8,40$ (US\$ 1.30); sputum culture, Cr\$22,00 (US\$ 3.50); treatment, Cr\$ 610,00 (US\$ 96.82). The data obtained were used in the mathematical model desbribed by Revelle and Male, but were modified by the author for calculating the cost per active case treated in all possible logical combinations of the available tests. The best pattern set down by economic, epidemiologic, and clinical criteria was the following: tuberculin testing of anyone resorting to the Health Unit; after that, smear examination only for the tuberculin reactors, complemented by culture examination of negative sputum; treatment of all the bacteriologic positive patients. In those Health Units where conditions allow roentgenphotography, this resource ought to be included in the above sequence between the tuberculin test and smear examination.

UNITERMS: Epidemiologic methods. Tuberculosis, pulmonary.

\section{REFERENCIAS BIBLIOGRAFICAS}

1. AHUMAdA. J. et al. Problemas conceptuales $y$ metodologicos de la programacion de la salud. Washington, D.C., Organizacion Panamericana de la Salud. 1965. (OPAS - Publ. cient., 111)

2. ANDREWS, R. H. \& RADHAKRISHNA, S. A comparison of two methods of sputum collection in the diagnosis of pulmonary tuberculosis. Tubercle, London, 40:155-62, 1959.

3. ARANTES, G. R, Planejamento anti-tuberculose: valor predictivo do teste tuberculinico negativo; influência da triagem tuberculinica no valor predictivo da abreugrafia. São Paulo, 1974. [Tese de Doutoramento - Facul 'ade de Saúde Pública da USP]. 
ARANTES, G. R. Aplicação de modelo matemático visando à escolha da melhor combinação de instrumentos para a descoberta e tratamento de casos de tuberculose pulmonar. Rev. Saúde públ., S. Paulo, 12:455-70, 1978.

4. ARANTES, G. R. Planejamento de atividades anti-tuberculose pelo método CENDES/OPS. Rev. Saúde públ., S. Paulo, 10:17-29, 1976.

5. ARANTES, G. R. \& TRIVELLATO, L. B. Cadastramento bacteriológico antituberculose: estudo preliminar para sua implantação em município do Estado de São Paulo. Rev. Saúde públ., S. Paulo, 10:167-76, 1976.

6. CAMPANHA NACIONAL CONTRA A TUBERCULOSE. Comissão Técnica. Prova tuberculínica em saúde pública. (2a. recomendação). Rev. Serv. nac. Tuberc., $12: 219-30,1968$.

7. CHAN, W. et al, Bacteriological measures for the detection of cases of pulmonary tuberculosis. Bull. Wld Hlth org., 45:551-8, 1971.

8. FAVERO, $M$. et al. Organização de um centro de informática hospitalar em nível local. Rev. paul. Hosp., 21:151-7, 1973.

9. FEREBEE, S. H. An epidemiological madel of tuberculosis in the United States. N. T. A, Bull., 53:4-7, 1967.

10. GRZYBOWSKI, S, et al. Contacts of cases of active pulmonary tuberculosis. Bull. int. Un. Tuberc., 50:90-106, 1975.

11. KECK, C. W. \& FOX, W. W. Tuberculosis control program in the Yungas area of Bolivia (final report). La Paz, Bolivia, Ministry of Public Health. Peace Corps, 1969.

12. KRIVINKA, $R$. et al. Epidemiological and clinical study of tuberculosis in the district of Kolin, Czechoslovakia (1965-1972). Bull. Wld Hlth Org., 51: $59-69,1974$

13. MINISTERIO DA SAƯDE. Divisão Nacional de Tuberculose. Ação anti-tuberculose em nivel nacional. Rio de Janeiro, 1971.

14. MINISTERIO DA SAỨDE. Divisão Nacional de Tuberculose. Normas para os laboratórios de baciloscopia de tuberculose. Rio de Janeiro, 1973.

15. MITCHISON, D. A. Examination of sputum by smear and culture in case finding. Bull. Int, Un. Tuberc., 41:139-47, 1968.
16. NATIONAL TUBERCULOSIS ASSOCIATION. Diagnostic standards and classification of tuberculosis. New York, 1961 .

17. PIOT, M. A. Strategy of BCG programmes under different socio-economic and epidemiological conditions including age-groups to be vaccinated and revaccination. Bull, int. Un. Tuberc., 41: 60-3, 1968.

18. RAO, K. P. \& NAGPAUL, D. R. Diagnostic bactériologique de la tuberculose pulmonaire: examen microscopique $d u$ crachat. Bull, int. Un. Tuberc., 44: 72-84, 1970.

19. REVELLE, C. \& MALE, J. Mathematical model for determining case finding and treatment activities in tuberculosis control programmes, Amer, Rev. resp. Dis., $102: 403-11,1970$.

20. SCHIMIDEK, $H$. $H$. et al. Pulmonary tuberculosis with normal chest radiography: report of eight cases. Carad. med. Ass, J., 97:178-80, 1967.

21. SENTIES, V. R. Programa de localizacion de casos de tuberculosis. In: Seminário Regional de Tuberculosis, 29, Bogotá, 1972. Washington, D. C., Organización Panamericana de la Salud, 1973. p. 48-55. (OPAS - Publ. clent., 265)

22, SHAW, J. B. \& WYNN-WILLIAMS, N. Infectivity of pulmonary tuberculosis in relation to sputum status. Amer. Rev. Tuberc., 69:724-32, 1954.

23. VAN GEUNS, $\mathbf{H}$, $A$, et al. Resuits of contact examination in Rotterdam, 1967-1969. Bull. int. Un. Tuberc., 50: 107-21, 1975 .

24. VECCHIo, T. J. Predictive value of a single diagnostic test in unselected populations. New Engl. J. Med., 274: 1171-3, 1966.

25. WAALER, H. T, \& PIOT, M. A. The use of an epidemiological model for estimating the effectiveness of tuberculosis control measures, Bull. Wld Flth Org., 41:75-93, 1969.

Recebido para publicaşão em 15/05/1978 Aprovado para publicasdo em 09/08/1978 
ARANTES, G. R. Aplicaçăo de modelo matemático visando à escolha da melhor combinação de instrumentos para a descoberta e tratamento de casos de tuberculose pulmonar. Rev. Sarude públ., S. Paulo, 12:455-70, 1978.

\section{ANEXO}

Fórmulas usadas para calcular o C CAT segundo as várias combinações lógicas possíveis dos instrumentos disponiveis para a descoberta dos casos, com os fatores de correção introduzidos (válidos apenas para a demanda estudada).

1 - baciloscopia $\longrightarrow$ tratamento

$$
\text { C.C.A.T. }=\frac{\left(\mathrm{C}_{\mathrm{b}} \times 0,042\right)+(\mathrm{F} \times \mathrm{B})}{\mathrm{B}}
$$

2 - baciloscopia $\longrightarrow$ tratamento

$$
\text { C.C.A.T. }=\frac{\left(C_{b} \times 0,042\right)+(F \times B)+C_{0}(0,042-B)+(F \times C)}{C+B}
$$

3 - baciloscopia $\longrightarrow$ tratamento

$$
\frac{\left(C_{b} \times 0,042\right)+C_{r}(1-B)+F(S+C+B)}{C+B}
$$

$4-$ baciloscopia $\longrightarrow$ tratamento

$$
\text { C.C.A.T. }=\frac{\left(C_{b} \times 0,042\right)+C_{r}(1-B)+C_{r}(S+C)+F(C+B)}{C+B}
$$

$$
\begin{aligned}
5-\text { baciloscopia } & \text { tratamento } \\
\text { C.C.A.T. } & =\frac{\left(C_{b} \times 0,042\right)+C_{t}(1-B)+F(1+S+C+B)}{C+B}
\end{aligned}
$$

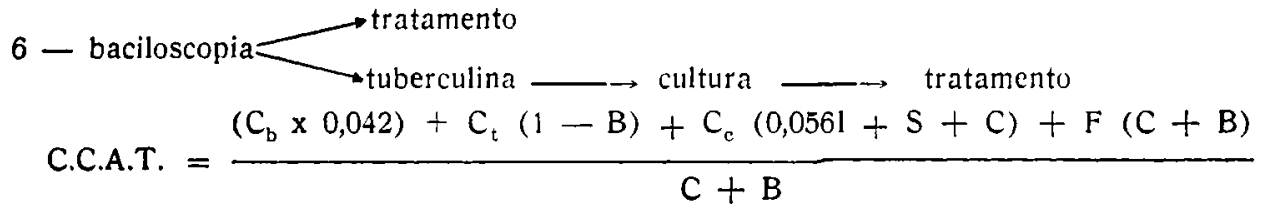

7 - baciloscopia $\longrightarrow$ tratamento

$$
\text { C.C.A.T. }=\frac{\left(C_{b} \times 0,042\right)+C_{t}(1-B)+C_{r}(I+S+C)+F(S+C+B)}{C+B}
$$


ARANTES, G. R. Aplicação de modelo matemático visando à escolha da melhor combinação de instrumentos para a descoberta e tratamento de casos de tuberculose pulmonar. Rev. Saúde públ., S. Paulo, 12:455-70, 1978.

$$
\begin{aligned}
& 8 \text { - baciloscopia } \longrightarrow \text { tratamento } \\
& \text { C.C.A.T. }=\frac{\left(\mathrm{C}_{\mathrm{b}} \times 0,042\right)+\mathrm{C}_{\mathrm{t}}(1-\mathrm{B})+\mathrm{C}_{\mathrm{r}}(\mathrm{I}+\mathrm{S}+\mathrm{C})+\mathrm{C}_{\mathrm{c}}(\mathrm{S}+\mathrm{C})+\mathrm{F}(\mathrm{C}+\mathrm{B})}{\mathrm{C}+\mathrm{B}}
\end{aligned}
$$

9 - abreugrafia tratamento

$$
\text { C.C.A.T. }=\frac{\left(\mathrm{C}_{\mathrm{r}} \times 1\right)+\mathrm{F}(\mathrm{S}+\mathrm{C}+\mathrm{B})}{\mathrm{C}+\mathrm{B}}
$$

$10-$ abreugrafia

$$
\text { tratamento }
$$

$$
\text { C.C.A.T. }=\frac{\left(C_{r} \times 1\right)+C_{c}(S+C+B)+F(C+B)}{C+B}
$$

$11-$ abreugrafia $\longrightarrow$ baciloscopia $\longrightarrow$ tratamento

$$
\text { C.C.A.T. }=\frac{\left(C_{r} \times 1\right)+C_{b}(S+C+B)+(F \times B)}{B}
$$

$$
\begin{aligned}
& 12 \text { - abreugrafia } \longrightarrow \text { baciloscopia } \longrightarrow \text { tratamento } \\
& \text { C.C.A.T. }=\frac{\left(\mathrm{C}_{\mathrm{r}} \times 1\right)+\mathrm{C}_{\mathrm{b}}(\mathrm{S}+\mathrm{C}+\mathrm{B})+\mathrm{C}_{\mathrm{c}}(\mathrm{S}+\mathrm{C})+\mathrm{F}(\mathrm{C}+\mathrm{B})}{\mathrm{C}+\mathrm{B}}
\end{aligned}
$$

$13-$ trberculina $\longrightarrow$ tratamento

$$
\text { C.C.A.T. }=\frac{\left(\mathrm{C}_{\mathrm{t}} \times 1\right)+\mathrm{F}(\mathrm{I}+\mathrm{S}+\mathrm{C}+\mathrm{B})}{\mathrm{C}+\mathrm{B}}
$$

14 - tuberculina $\longrightarrow$ cultura $\longrightarrow$ tratamento

$$
\text { C.C.A.T. }=\frac{\left(C_{t} \times 1\right)+C_{c}(0,0561+S+C+B)+F(C+B)}{C+B}
$$

15 - tuberculina $\longrightarrow$ abreugrafia $\longrightarrow$ tratamento

$$
\text { C.C.A.T. }=\frac{\left(C_{t} \times 1\right)+C_{r}(I+S+C+B)+F(S+C+B)}{C+B}
$$


ARANTES, G. R. Aplicaçăo de modelo matemático visando à escolha da melhor combinação de instrumentos para a descoberta e tratamento de casos de tuberculose pulmonar. Rev. Saúde públ., S. Paulo, 12:455-70, 1978.

$$
\begin{aligned}
& 16-\text { tuberculina } \longrightarrow \text { abreugrafia } \overrightarrow{\left(C_{t} \times 1\right)+C_{r}(I+S+C+B)+C_{c}(S+C+B)+F(C+B)} \\
& \text { C.C.A.T. }=\frac{\text { cultura } \rightarrow B}{17-\text { tratamento }} \\
& \text { C.C.A.T. }=\frac{\left(C_{t} \times 1\right)+C_{r}(I+S+C+B)+C_{b}(S+C+B)+(F \times B)}{B} \\
& 18-\text { tuberculina } \rightarrow \text { abreugrafia } \rightarrow \text { baciloscopia } \rightarrow \text { tratamento } \rightarrow \text { tratamento } \\
& \text { C.C.A.T. }=\frac{\left(C_{t} \times 1\right)+C_{r}(1+S+C+B)+C_{b}(S+C+B)+C_{c}(S+C)+F(C+B)}{C+B}
\end{aligned}
$$

19 - tuberculina tratamento

$$
\text { C.C.A.T. }=\frac{\left(C_{l} \times 1\right)+C_{b}(0,0561+S}{B}
$$

$$
\left(C_{t} \times 1\right)+C_{b}(0,0561+S+C+B)+(F \times B)
$$

$$
\begin{aligned}
& 20-\text { tuberculina } \longrightarrow \text { baciloscopia } \longrightarrow \text { tratamento } \\
& \text { C.C.A.T. }=\frac{\left(C_{t} \times 1\right)+C_{b}(0,056 \mathrm{I}+\mathrm{S}+\mathrm{C}+\mathrm{B})+\mathrm{C}_{\mathrm{c}}(0,056 \mathrm{I}+\mathrm{S}+\mathrm{C})+\mathrm{F}(\mathrm{C}+\mathrm{B})}{\mathrm{C}+\mathrm{B}}
\end{aligned}
$$

$$
\begin{aligned}
& 21-\text { tuberculina } \longrightarrow \text { baciloscopia } \longrightarrow \text { tratamento } \\
& \text { C.C.A.T. }=\frac{\left(C_{t} \times 1\right)+C_{b}(0,056 I+S+C+B)+C_{r}(I+S+C)+F(S+C+B)}{C+B}
\end{aligned}
$$

22 - tuberculina $\longrightarrow$ baciloscopia $\longrightarrow$ tratamento

$$
\left(C_{t} \times 1\right)+C_{b}(0,056 I+S+C+B)+C_{r}(I+S+C)+C_{c}(S+C)+F(C+B)
$$

C.C.A.T. $=$

$$
C+B
$$

23 - cultura tratamento

$$
\text { C.C.A.T. }=\frac{\left(C_{c} \times 0,042\right)+F(C+B)}{C+B}
$$

